\title{
Targeted exhaled breath analysis for detection of Pseudomonas aeruginosa in cystic fibrosis patients
}

\author{
Renate Kos ${ }^{a}$, Paul Brinkman ${ }^{a}$, Anne H. Neerincx ${ }^{a}$, Tamara Paff ${ }^{b}$, Marije G. Gerritsen ${ }^{a}$, \\ Ariana Lammers ${ }^{a}$, Aletta D. Kraneveld ${ }^{\text {c,d }}$, Harry G.M. Heijerman ${ }^{\mathrm{e}}$, Hettie M. Janssens ${ }^{\mathrm{f}}$, \\ Jane C. Davies ${ }^{\text {g,h }}$, Christof J. Majoor ${ }^{\mathrm{a}}$, Els J. Weersink ${ }^{\mathrm{a}}$, Peter J. Sterk ${ }^{\mathrm{a}}$, Eric G. Haarman ${ }^{\mathrm{b}}$, \\ Lieuwe D. Bos ${ }^{\mathrm{a}, \mathrm{i}}$, Anke H. Maitland-van der Zee ${ }^{\mathrm{a}, \mathrm{b}, *}$, on behalf of the Amsterdam \\ Mucociliary Clearance Disease (AMCD) Research Group and the Amsterdam UMC Breath \\ Research Group
}

${ }^{a}$ Department Respiratory Medicine, Amsterdam University Medical Centres - loc. AMC, University of Amsterdam, Amsterdam, Netherlands

${ }^{\mathrm{b}}$ Department Paediatric Respiratory Medicine and Allergy, Emma Children's Hospital, Amsterdam University Medical Centres, Amsterdam, the Netherlands

${ }^{c}$ Division of Pharmacology, Utrecht Institute for Pharmaceutical Sciences, Faculty of Science, Utrecht University, Utrecht, Netherlands

${ }^{\mathrm{d}}$ Institute for Risk Assessment Sciences, Faculty of Veterinary Medicine, Utrecht University, Utrecht, Netherlands

e Department Respiratory Medicine, University Medical Centre, Utrecht, Netherlands

${ }_{\mathrm{f}}^{\mathrm{f}}$ Department of Paediatrics, Division Respiratory Medicine and Allergology, Erasmus MC/Sophia Children's Hospital, University Medical Centre, Rotterdam, Netherlands

${ }^{g}$ National Heart and Lung Institute, Imperial College London, London, United Kingdom

${ }^{\mathrm{h}}$ Department of Paediatric Respiratory Medicine, Royal Brompton Hospital, London, United Kingdom

i Department of Intensive Care, Amsterdam University Medical Centres, University of Amsterdam, Netherlands

\section{A R T I C L E I N F O}

Article history:

Received 10 November 2020

Revised 8 April 2021

Accepted 23 April 2021

Available online 18 May 2021

\section{Keywords:}

Cystic fibrosis

Pseudomonas aeruginosa

Exhaled biomarkers

$\mathrm{VOC}$

\begin{abstract}
A B S T R A C T
Background: Pseudomonas aeruginosa $(P A)$ is an important respiratory pathogen for cystic fibrosis (CF) patients. Routine microbiology surveillance is time-consuming, and is best performed on expectorated sputum. As alternative, volatile organic compounds (VOCs) may be indicative of $P A$ colonisation. In this study, we aimed to identify VOCs associated with $P A$ in literature and perform targeted exhaled breath analysis to recognize $P A$ positive $C F$ patients non-invasively.

Methods: This study consisted of 1) a literature review to select VOCs of interest, and 2) a cross-sectional CF study. Definitions used: A) PA positive, $P A$ culture at visit/chronically; B) $P A$ free, no $P A$ culture in $\geq 12$ months. Exhaled VOCs were identified via quadrupole MS. The primary endpoint was the area under the receiver operating characteristics curve (AUROCC) of individual VOCs as well as combined VOCs against PA culture.

Results: 241 VOCs were identified in literature, of which 56 were further evaluated, and 13 could be detected in exhaled breath in our cohort. Exhaled breath of 25 pediatric and 28 adult CF patients, PA positive $(n=16)$ and free $(n=28)$ was available. $3 / 13$ VOCs were significantly $(\mathrm{p}<0.05)$ different between PA groups in children; none were in adults. Notably, a composite model based on 5 or 1 VOC(s) showed an AUROCC of 0.86 ( $\mathrm{CI} 0.71-1.0)$ and 0.87 ( $\mathrm{Cl} 0.72-1.0)$ for adults and children, respectively.

Conclusions: Targeted VOC analysis appears to discriminate children and adults with and without $P A$ positive cultures with clinically acceptable sensitivity values.
\end{abstract}

(C) 2021 The Author(s). Published by Elsevier B.V. on behalf of European Cystic Fibrosis Society. This is an open access article under the CC BY license (http://creativecommons.org/licenses/by/4.0/)
Substantial contributions to the conception or design of the work: TP, EW, PS, EH, LB, AM. The acquisition and analysis: RK, TP, MG, CM, EW, EH, PB, LB. Interpretation of data for the work: RK, PB, AN, AL, AK, HH, HJ, JCD, PS, LB, AM. Drafting the work or revising it critically for important intellectual content: ALL. Final approval of the version to be published: ALL. Agreement to be accountable: ALL.

the Members of the Amsterdam mucociliary clearance disease research group: J. Altenburg, N.W. Rutjes, S.W.J. Terheggen-Lagro, D. Seljogi, E.M. Kemper, R. Lutter, 


\section{Introduction}

Cystic fibrosis (CF) is a recessively inherited, progressive multiorgan disease that results in infection of the respiratory tract by pathogens such as Pseudomonas aeruginosa (PA). Infection is associated with increased morbidity, greater decline in lung function, and increased time in hospital [1-6]. Eradication of PA should be initiated swiftly, but monitoring of colonisation is based on microbial sputum cultures, which frequently cannot be obtained in young CF patients. Alternatives to sputum cultures are either less sensitive (throat swabs) or highly invasive (bronchoscopy) [7]. Moreover, the result of sputum cultures take one week and lack in sensitivity, delaying prompt treatment of infections [8,9].

Exhaled breath analysis provides a non-invasive alternative for microbial cultures. Volatile organic compounds (VOCs) in exhaled breath originate from different processes: 1) host metabolism in the lung and elsewhere, 2) microbial metabolism and 3) foreign sources such as contamination via inhaled air or the oropharynx [10]. Several studies have suggested that VOCs allow distinguishing between bacterial species in vitro and also diagnosing infected humans [11-15]. However, studies do not show consistent results due to a plethora of confounders [16]. While unrelated metabolomic processes affect human in vivo studies, culture conditions lack any signal from host-responses. Therefore, despite the abundant VOC presence in exhaled breath, no exhaled breath test is currently implemented in clinic.

The next step forward for exhaled breath biomarkers would be to transcend from discovery science into hypothesis driven targeted analysis. Combining current knowledge on VOCs from both in vitro cultures and in/ex vivo human studies, decreases the chance of falsely detecting associations [17]. Using this as a basis for targeted analysis in real-life patient samples, we can potentially validate exhaled breath biomarkers for pathogen detection in CF patients.

Our central hypothesis is that VOCs of PA can be detected in the breath of patients with $\mathrm{CF}$ and can be used to accurately identify colonised patients with a high sensitivity. To answer this hypothesis, we conducted a two-step analysis. First, we created an overview of VOCs that have been associated with the presence of $P A$ in both in vitro cultures and human studies to increase the $a$ priori probability of a direct biochemical link between Pseudomonas and VOCs. Second, we analysed the breath of patients with CF for the presence of a subset of these VOCs and evaluated the accuracy for detection of $P A$ infection in sputum or cough swabs of the same patients.

\section{Methods}

\subsection{Systematic literature review}

A broad systematic search in the PubMed Central library was performed by RK, using the following terms: 'Pseudomonas aeruginosa' AND 'volatile' AND ('mass spectrometry' OR 'spectroscopy'). Selection for full-text examination was based on the title and/or abstract suggesting the measurement of VOCs in relation to PA. If during full-text examination an article included a summary of

S.J. Vijverberg, S.E.M. Vonk, J.W.F. Dagelet, D.W. Fenn, N. Adriaens, R. Lub, M. van Brederode, L. van der Schaaf, M. Verkleij, N.A. van Gilst, G.H. Hofsteenge, C.L.H. Brackel, P. Lakeman, I.C.M. Bon, S.P.M. Tanner.

* Members of the Amsterdam UMC breath research group: C. Longo, A. Sinha, D. Fenn, L.B. Richards, J.M.H. van Bragt, M. I. Abdel-Aziz, J.W.F. Dagelet, S.J.A. Lone-Latif, M.J. Schultz, M.R. Smit, L.A. Hagens.

* Corresponding author at: Meibergdreef 9, 1105 AZ, Amsterdam, F5-257, Netherlands.

E-mail address: a.h.maitland@amsterdamumc.nl (A.H. Maitland-van der Zee). detected VOCs released in the presence of $P A$, the article was included. There was no restriction to in vitro or human studies, nor was there a restriction in publication date. Double publications of the same data and publications in a language other than English were disregarded. All VOCs were summarized based on the following molecular structures (adapted from Hakim et al. [18]): hydrocarbons, alcohols, acids, aldehydes, ketones, cyclic compounds, esters, S-containing, and $\mathrm{N}$-containing.

\subsection{Clinical study design and subjects}

\subsubsection{Design and ethical considerations}

We used data of the first visit of a longitudinal study in which children and adults with CF were followed for one year. The study was approved by the Institutional Research Board of the Academic Medical Centre (B2012500). Patients were recruited in the Amsterdam Medical Centers between 2013-2014. Written informed consent was obtained from all patients, or in the case of minors, from their representatives, before data collection.

\subsubsection{Population and definitions}

Patients were recruited at the outpatient clinic. Inclusion criteria were (i) CF diagnosis based on clinical symptoms in combination with an abnormal sweat test (chloride $>60 \mathrm{mmol} / \mathrm{l}$ ) and/or identification of mutations in both alleles of the cystic fibrosis transmembrane conductance regulator (CFTR) gene, [19] (ii) stable respiratory disease for at least 6 weeks (as determined by the treating physician) and (iii) ability to perform lung function. Exclusion criteria were mental retardation, CF related diabetes, technically unsatisfactory performance of measurements, on the waiting list for lung transplantation and participation in other clinical studies.

Patients were considered $P A$ positive if their $P A$ culture was positive at the visit, or were chronically $P A$ infected. Chronic $P A$ infection was defined as more than $50 \%$ of all available sputum/swab cultures were $P A$ positive during the 12 months before inclusion (with a minimum of 2 positive samples); Free of $P A$ was defined as no growth of $P A$ during the 12 months before inclusion [20]. Patients who were intermittently infected in the previous year, but did not test positive at this visit were considered 'ambiguous' and were excluded.

\subsubsection{Measurements}

Sputum and cough swab cultures and standardized lung function test were part of routine clinical CF care and the results were noted in a case record form. Data on sputum cultures were collected from the electronic patient files from one year prior to inclusion.

Exhaled breath was collected as described by Van der Schee et al. [21]. In short, after a 5-minute wash-out period in which patients breathed through a mouthpiece connected to a VOC filter (A1-filter, North, N7500-1U), while nasal breathing was circumvented by a nose clip. Thereafter, patients performed an inspiratory capacity manoeuvre and exhaled into a nalophan bag after connection to the mouthpiece. Within 10 minutes after collection, $500 \mathrm{~mL}$ of exhaled air was sampled through a thermal desorption tube (Tenax GR 60/80 Interscience, Breda, The Netherlands) at a flow of $250 \mathrm{~mL} / \mathrm{min}$ using a peristaltic pump, to collect, transport and store the expired VOCs.

\subsection{Exhaled breath analysis}

Samples were analysed by gas chromatography - mass spectrometry (GC-MS) as previously described by Van der Schee et al. [21]. In short, the VOCs were released from the sorbent material by heating the tube in a thermal desorption unit 
(TD100, Markes, Cincinnati, Ohio, USA) to $280^{\circ} \mathrm{C}$ for 15 minutes with a flow of $30 \mathrm{ml} / \mathrm{min}$. Subsequently, compounds were cold trapped at $10^{\circ} \mathrm{C}$, rapidly heated to $300^{\circ} \mathrm{C}$ for 1 minute and split less injected at $180^{\circ} \mathrm{C}$ through a transfer line onto an Intercap $5 \mathrm{MS} /$ Sil GC column $(30 \mathrm{~m}$ ", $0.25 \mathrm{~mm}$ ID, $1 \mu \mathrm{m}$ film thickness, 1,4bis(dimethylsiloxy)phenylene dimethyl polysiloxane, Restek, Breda, The Netherlands) at $1.2 \mathrm{ml} / \mathrm{min}$. Ionization of the molecules was done using electron ionization at $70 \mathrm{eV}$ after which the fragment ions were detected using a quadrupole mass-spectrometer (GCMSGP2010, Shimadzu, Den Bosch, The Netherlands). The scan range was 37-300 Da. VOCs of interest as obtained by the literature study were identified manually in the GC-MS spectra by matching in the NIST-library and by retention time. The combination of a unique mass-to-charge ratio and retention time was used for subsequent statistical analysis. Furthermore, analytical standards (HPLC grade $\geq 99.7 \%$, Honeywell Riedel-de Haën Chromasolv ${ }^{\mathrm{TM}}$, Landsmeer, The Netherlands) were injected into the GC-MS to confirm the retention time and fragmentation of compounds of interest.

\subsection{Endpoint}

The primary endpoint of this study was the diagnostic accuracy for $P A$ infection detection using the VOCs that were associated with $P A$ in literature and were identified in the breath of CF patients, who are considered as $P A$ positive by airway cultures. This was reported in area under the receiving operator characteristics curve (AUROCC) with sensitivity and specificity at the cut-off closest to the top-left corner. This comparison was performed for adults and children separately.

\subsection{Statistical analysis}

Statistical analysis was performed in RStudio V1.2.1335 (RStudio Inc., Boston, United States of America). Descriptive statistics were compared via T-test, Mann-Whitney $\mathrm{U}$ analysis, or $\mathrm{Chi}^{2}$ test. Means of VOCs were compared by Mann-Whitney U tests.

VOC data was log-scaled to stabilize the variance of results [22]. Odds ratios of VOCs were determined by univariate binary logistic regression and $95 \%$ confidence intervals $(95 \% \mathrm{CI})$ were established. A receiver operating characteristics curve was constructed and area under the curve (AUROCC) with 95\% CI was reported. A forward stepwise regression was performed to create a multivariate model: starting with the most significant variable (VOC) until no significant model improvement was reached by adding another variable. All models were tested by a leave-one-out cross validation.

\section{Results}

\subsection{Systematic review}

The PubMed Central library search resulted in 314 articles, out of these articles 64 were selected for full text analysis, 40 articles published between 1977 and 2019 were eventually included in this study, Figure S1. From all included articles, a list of 241 chemical compounds (Tables S2-S10) was constructed. 56 compounds were considered VOCs of interest based on multiple consistent notations in literature, Figure S2.

\subsection{Targeted analysis of human breath}

\subsubsection{Patient characteristics}

In total 53 patients ( 25 children) were included in the study cohort; two were excluded from analysis due to missing GCMS data. Patient characteristics are described in Table S11. Cultures of Haemophilus influenza and Staphylococcus aureus were more prevalent in children, whereas other cultures were less prevalent in children (Table S11). 13 adults and 15 children were classified as PA

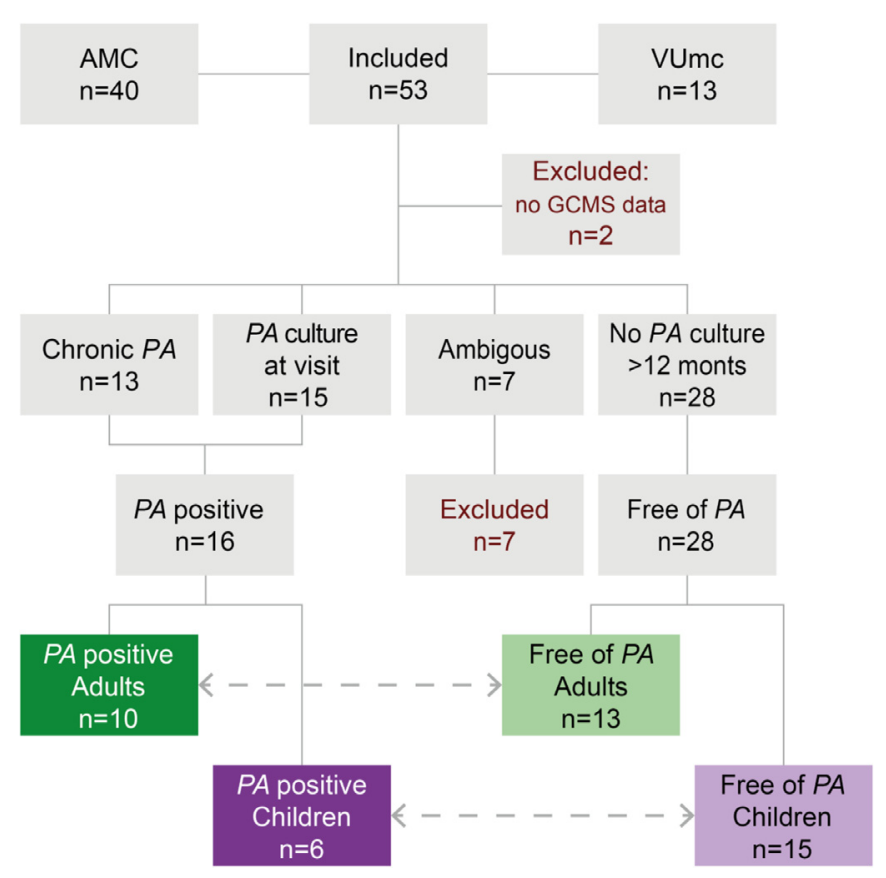

Fig. 1. Schematic overview of patient selection. Arrows indicate groups compared during statistical analysis. GC-MS: gas chromatography-mass spectrometry, PA, Pseudomonas aeruginosa.

free; 10 adults and 6 children were classified as PA positive, 7 patients were excluded based on ambiguous PA status (positive tests over the last year, but neither chronically infected or positive at time of breath analysis) (Fig. 1). Patient characteristics are described in Table 1. Lung function was lower $(\mathrm{p}<0.01)$ in $P A$ positive adults. Children frequently showed co-cultures with Staphylococcus aureus; adults show a more diverse profile of co-cultures with a variety of pathogens.

\subsubsection{Detection of VOCs}

Exhaled breath was analysed for 51 out of 53 included patients. After deconvolution, 2024 ion-fragments were quantified. 13 of the 56 VOCs of interest were found in the exhaled breath of the cohort (Table S12). 3 out of 13 VOCs were significantly different between children and adults, Table S13.

\subsubsection{VOC correlation with PA presence}

Of the 13 VOCs, 3 were significantly negatively associated with $P A$ presence in children: 2-butanone (odds ratio: $0.05,95 \%-\mathrm{CI}$ : 0.00-0.66), ethyl acetate (odds ratio: $0.27,95 \%-\mathrm{CI}: 0.05-0.79$ ), and heptane,2,4-dimethyl (odds ratio: 0.5, 95\%-CI: 0.21-0.97); Table 2 and Fig. S3. None of the individual VOCs was significantly associated with PA presence in adults. The AUROCC ranged from 0.510.87 for children and $0.51-0.67$ for adults. Ethyl acetate showed the highest predictive value for $P A$ in both children and adults despite the insignificant difference between groups, Fig. 2 .

\subsubsection{Multivariate regression model}

Despite that no individual VOC was a significantly associated with $P A$ presence in adults, the multivariate logistic regression model reached an optimum at five VOCs. The composite model for adults included ethyl acetate, limonene, 2-pentanone 3-methyl, toluene, and 2-butanone and had a $78.3 \%$ accuracy during crossvalidation and an AUROCC of 0.86 (95\% CI: 0.70-1.0, Fig. 2). The closest to top-left cut-off resulted in $100 \%$ specificity and $70 \%$ sensitivity.

Multivariate analysis did not result in increased accuracy over the univariate analysis for the identification of $P A$ presence in chil- 
Table 1

Patient characteristics. Culture data and medication usage is described as is at the time of the visit. The p-value was obtained after comparison between PA status by T-test, Mann Whitney-U test or $\mathrm{Chi}^{2}$ test, if applicable. Bold: Significantly different, $\mathrm{p}$-value $<0.5$

\begin{tabular}{|c|c|c|c|c|c|c|}
\hline & \multicolumn{3}{|l|}{ Adults } & \multicolumn{3}{|l|}{ Children } \\
\hline & $\begin{array}{l}\text { Free of } P A \\
\text { Mean (SD) /N (\%) }\end{array}$ & $\begin{array}{l}\text { PA positive } \\
\text { Mean (SD) /N (\%) }\end{array}$ & $P$ val. & $\begin{array}{l}\text { Free of } P A \\
\text { Mean (SD) /N (\%) }\end{array}$ & $\begin{array}{l}\text { PA positive } \\
\text { Mean (SD) /N (\%) }\end{array}$ & P val. \\
\hline Patients [N] & 13 & 10 & - & 15 & 6 & - \\
\hline Gender - Female [N (\%)] & $5(38.5)$ & $4(40)$ & 0.94 & $7(46.7)$ & $3(50)$ & 0.70 \\
\hline Age [Mean (SD)] & $27.0(12.9)$ & $27.6(6.7)$ & 0.39 & $9.5(2.7)$ & $12.8(3.4)$ & 0.39 \\
\hline BMI kg/m2 [Mean (SD)] & $24.0(3.3)$ & $21.3(2.9)$ & 0.06 & $17.1(2.3)$ & $18.2(2.9)$ & 0.30 \\
\hline FVC \% of Predicted [Mean (SD)] & $106.4(19.6)$ & $73.2(12.6)$ & 0.00 & $103.3(15.4$ & $95(17.1)$ & 0.10 \\
\hline FEV1 \% of Predicted [Mean (SD)] & $91.7(25.6)$ & $48.5(16.3)$ & 0.00 & 96.7 17.1) & 92 18.0) & 0.25 \\
\hline Diabetes Mellitus [N (\%)] & $0(0)$ & $0(0)$ & - & $0(0)$ & $0(0)$ & - \\
\hline Delta F508 Homozygote [N (\%)] & $4(30.8)$ & $5(50)$ & 0.49 & $9(60)$ & $3(50)$ & 0.64 \\
\hline Smoking [N (\%)] & & & - & & & - \\
\hline Current Smoker & $0(0)$ & $0(0)$ & - & $0(0)$ & $0(0)$ & - \\
\hline History of Smoking & $1(20)$ & $1(7.7)$ & - & $0(0)$ & $0(0)$ & - \\
\hline Culture - Cough swabs [N (\%)] & $4(19)$ & $0(0)$ & 0.12 & $12(86)$ & $2(40)$ & 0.16 \\
\hline Culture Positive [N (\%)] & & & - & & & - \\
\hline Achromabacter xylosoxidans & $2(15.4)$ & $0(0)$ & 0.19 & $0(0)$ & $0(0)$ & - \\
\hline Aspergillus fumigatus & $1(17.9)$ & $2(40)$ & 0.38 & $0(0)$ & $0(0)$ & - \\
\hline Burkholderia cepacia & $0(0)$ & $1(10)$ & - & $0(0)$ & $0(0)$ & - \\
\hline Haemophilus influenzae & $0(0)$ & $2(20)$ & - & $5(33.3)$ & $0(0)$ & 0.18 \\
\hline Staphylococcus aureus & $4(30.8)$ & $5(50)$ & 0.35 & $10(66.7)$ & $3(50)$ & 0.35 \\
\hline Stenotrophomonas maltophilia & $2(15.4)$ & $1(10)$ & 0.70 & $0(0)$ & $0(0)$ & - \\
\hline Bronchodilator [N (\%)] & $8(61.5)$ & $3(30)$ & 0.13 & $6(40)$ & $4(66.7)$ & 0.15 \\
\hline Inhaled corticosteroids [N (\%)] & $8(61.5)$ & $4(40)$ & 0.31 & $2(13.3)$ & $2(33.3)$ & 0.23 \\
\hline Maintenance antibiotics [N (\%)] & & & - & & & - \\
\hline Oral Antibiotics & $6(46.2)$ & $7(70)$ & 0.25 & $\mathbf{0}(\mathbf{0})$ & $2(33.3)$ & 0.01 \\
\hline Inhaled Antibiotics & $6(46.2)$ & $8(80)$ & 0.10 & $1(6.7)$ & $3(50)$ & 0.01 \\
\hline Antibiotics course $[\mathrm{N}(\%)]$ & & & - & & & - \\
\hline Oral Antibiotics & $0(0)$ & $3(30)$ & 0.10 & $0(0)$ & 2 & 0.11 \\
\hline Inhaled Antibiotics & $0(0)$ & $0(0)$ & - & $0(0)$ & $0(0)$ & - \\
\hline IV Antibiotics & $0(0)$ & $2(20)$ & 0.19 & $0(0)$ & 1 & 0.95 \\
\hline
\end{tabular}

BMI: Body Mass Index, FEV1: Forced Expiratory Volume in 1 second, FVC: Forced Vital Capacity, PA: Pseudomonas aeruginosa, P val: p-value, SD: standard deviation.

Table 2

Comparison of CF patient free of PA and PA positive. P-value as per Mann-Whitney U test. Odds ratios as obtained by logistic regression. Bold: Significantly different, p-value $<0.5$; Underlined, integrated in the composite model for adults

\begin{tabular}{|c|c|c|c|c|c|c|c|c|}
\hline & \multicolumn{4}{|c|}{ Adults } & \multicolumn{4}{|c|}{ Children } \\
\hline & OR & $\begin{array}{l}\text { 95\% CI } \\
\text { Low. - Up. }\end{array}$ & AUROCC & P val. & OR & $\begin{array}{l}\text { 95\% CI } \\
\text { Low. - Up. }\end{array}$ & AUROCC & P val. \\
\hline 1-propanol, 2-methyl (isobutanol) & 1.16 & $0.51-2.87$ & 0.52 & 0.88 & 1.25 & $0.57-2.82$ & 0.61 & 0.5 \\
\hline 2-butanone & 0.79 & $0.19-3.15$ & 0.65 & 0.23 & 0.05 & $0.00-0.66$ & 0.81 & 0.04 \\
\hline$\overline{2 \text {-pentanone }}$ & 0.99 & $0.47-2.18$ & 0.52 & 0.88 & 1.06 & $0.30-4.24$ & 0.56 & 0.75 \\
\hline$\overline{\text { 2-pentanone, 3-methyl }}$ & 0.75 & $0.44-1.15$ & 0.65 & 0.26 & 1.3 & $0.75-2.63$ & 0.60 & 0.56 \\
\hline 2,4-dimethyl-1-heptene & 1.12 & $0.59-2.21$ & 0.55 & 0.74 & 0.35 & $0.08-1.02$ & 0.79 & 0.07 \\
\hline Acetone & 0.96 & $0.25-3.76$ & 0.52 & 0.93 & 0.47 & $0.08-2.59$ & 0.60 & 0.56 \\
\hline Disulfide, dimethyl (DMDS) & 0.93 & $0.54-1.55$ & 0.58 & 0.56 & 0.93 & $0.43-1.84$ & 0.51 & 0.96 \\
\hline Dodecane & 1.03 & $0.13-8.98$ & 0.55 & 0.69 & 0.54 & $0.10-2.31$ & 0.70 & 0.22 \\
\hline Ethanol & 0.90 & $0.27-2.81$ & 0.52 & 0.93 & 1.1 & $0.45-3.15$ & 0.56 & 0.75 \\
\hline Ethyl acetate & 2.15 & $0.84-7.59$ & 0.67 & 0.19 & 0.27 & $0.05-0.79$ & 0.87 & 0.01 \\
\hline Heptane, 2,4-dimethyl & 1.20 & $0.62-2.53$ & 0.56 & 0.65 & 0.5 & $0.21-0.97$ & 0.81 & 0.04 \\
\hline Limonene & 0.52 & $0.15-1.43$ & 0.66 & 0.21 & 0.57 & $0.19-1.35$ & 0.63 & 0.44 \\
\hline Toluene & 1.12 & $0.75-1.91$ & 0.55 & 0.69 & 0.07 & $0.00-1.48$ & 0.73 & 0.16 \\
\hline
\end{tabular}

OR: odds ratio; 95\% CI: 95\% confidence interval; AUROCC: area under the receiver operating characteristics curve, P val: p-value.

dren. The univariate ethyl acetate model had an AUROCC of 0.87 (95\% CI: 0.71-1.0), with a 78.9\% accuracy during cross-validation, Fig. 2. The closest to top-left cut-off resulted in a $71.4 \%$ specificity and a $100 \%$ sensitivity.

\section{Discussion}

This study provides evidence that detection of $P A$ airway infections in CF patients is feasible using targeted VOC analysis, with, at least in children, clinically acceptable sensitivity and specificity. A large set of previously in literature reported VOCs was reduced to a small number of VOCs identified in exhaled breath of $P A$ infected CF patients. Importantly, a good diagnostic accuracy of ethyl acetate was found in children. This marker advances as the most important candidate for clinical implementation.

The literature search of a wide range of studies showed that many VOCs were either only detected in vitro, or in human studies (in/ex vivo). This may be explained by the fact that some metabolites are merely produced in vitro in non-clinically related processes. Culture medium influences VOC production considerably $[22,23]$. The metabolic composition of blood agar, a typical culture ground for bacteria, is very unlike respiratory epithelium. In vivo, a more complex system affects exhaled breath, including interactions between host and pathogen and interactions between polymicrobial populations [23,24]. In line with this, Zhu et al., [25] demonstrated that VOC profiles of $P A$ and SA cultures only showed a 25- 
A.

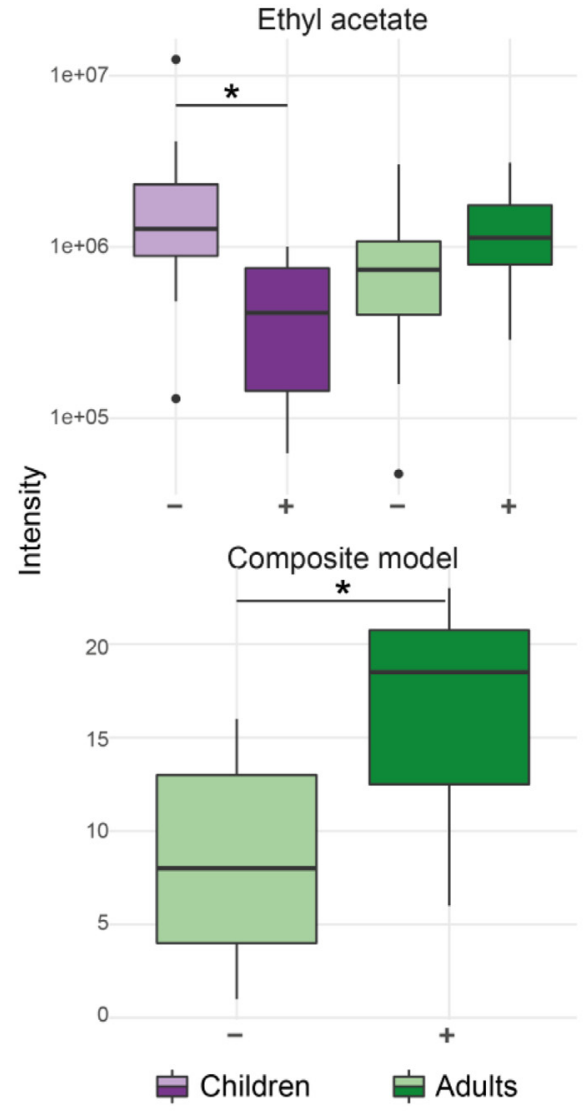

B.

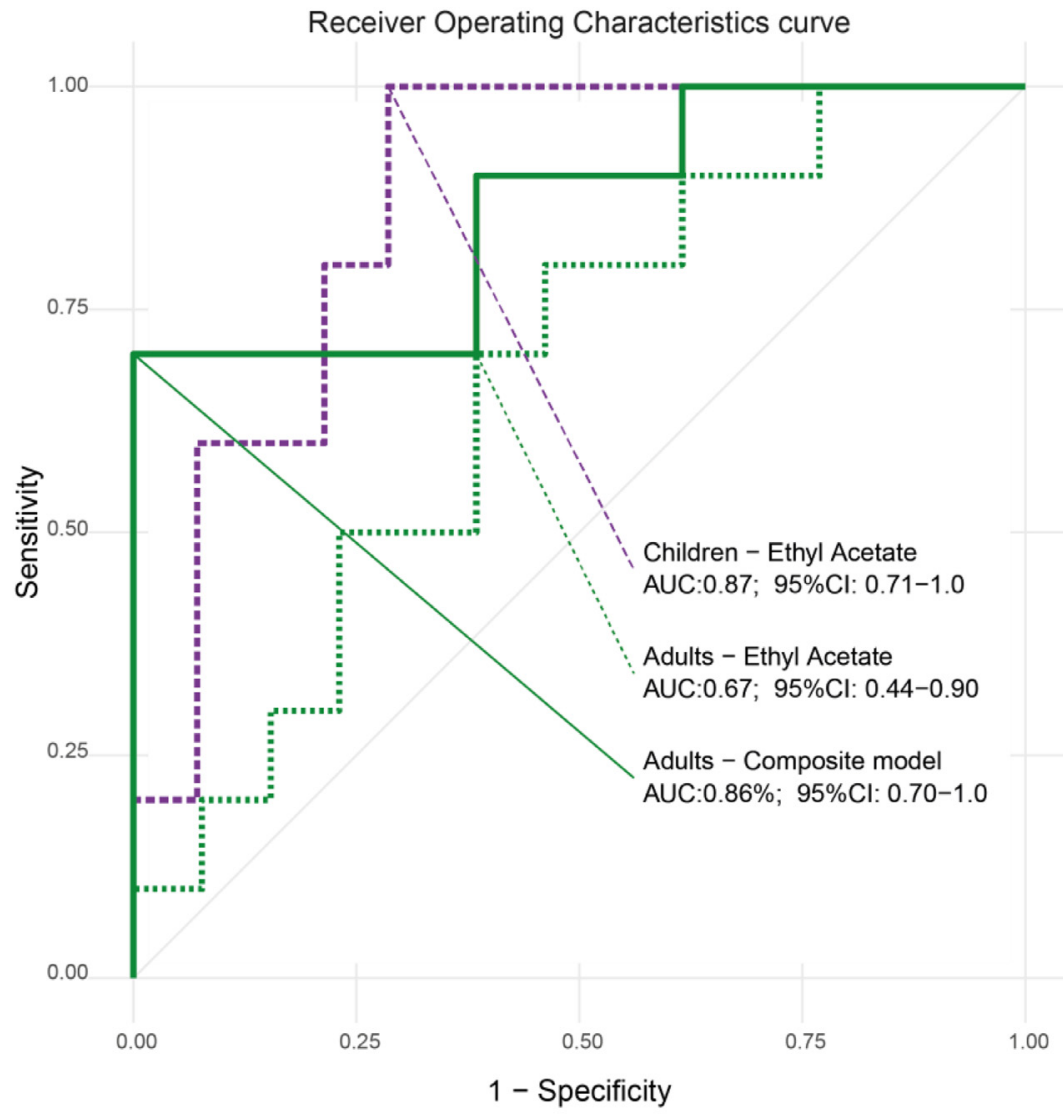

C.
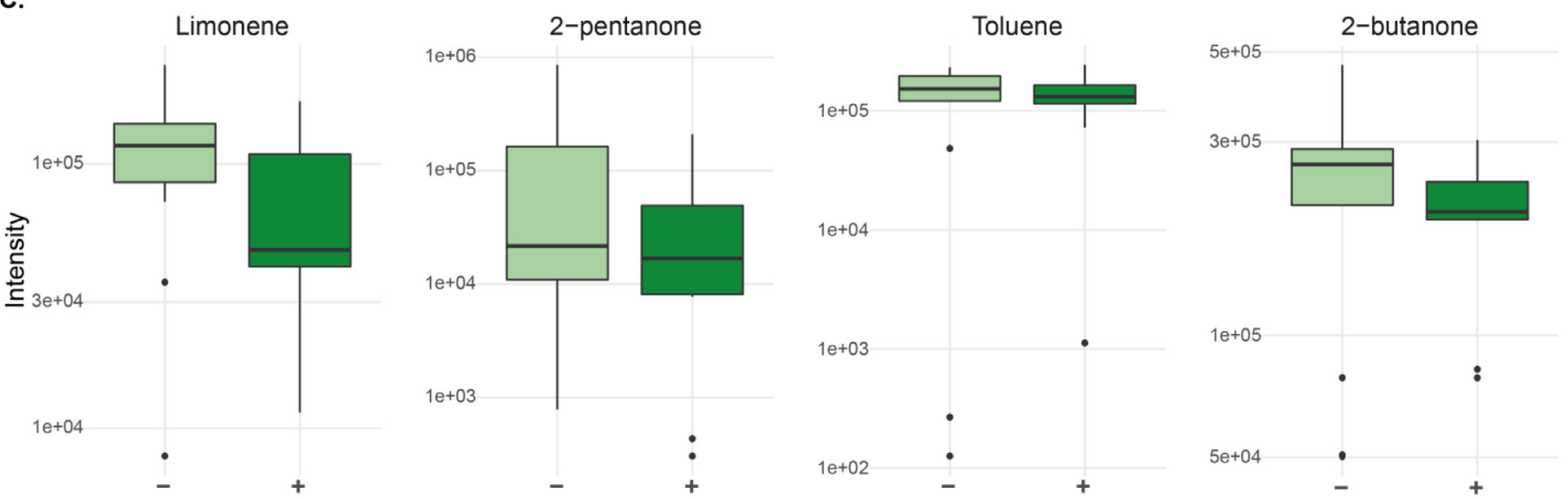

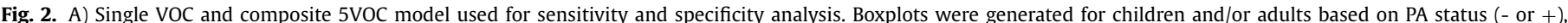

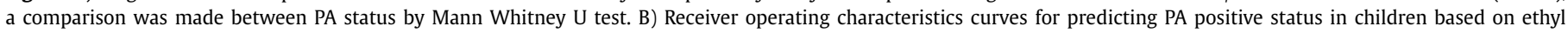

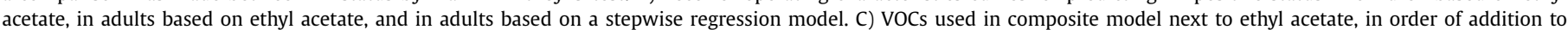
the model. AUC, area under the curve; EA, Ethyl Acetate, SRM, Stepwise Regression Model, 95\% CI, 95\% confidence interval.

$34 \%$ similarity between in vitro and in vivo results. Thus, a combination of methods likely yields compounds of the highest clinical relevance.

Moreover, we detected 13 out of the 56 VOCs identified in our GC-MS dataset, all 13 were previously reported in in/ex vivo studies. Coincidently, a significant number of VOC's of interest was not found in this study. This may be attributed to differences in detection method of VOCs: (1) sample collection procedures, (2) sorbent material for trapping of the VOCs, (3) gas chromatography choices (solid phase of the column, temperature programme), (4) resolution and sensitivity of the mass spectrometer. The combination of trapping on Tenax sorbent material and the separation on a nonpolar column does not allow for adequate separation of some of the nitrogen and sulphide containing molecules of interest, these require a different experimental setup.

Two ketones, part of the composite model for PA detection in adults, 2-butanone and 2-pentanone, were inversely related to the presence of $P A$. This conflicts with previously published data by 
Bean et al. that showed a positively correlation with PA presence in cultured clinical isolates from non-respiratory infections (e.g. eye, blood, skin), [26,27] concordant with several in vitro studies.[2831] The positive correlations were thus found in non-respiratory settings. Goeminne et al., supported our findings of a negative correlation in their study of respiratory $P A$ infections [14]. Therefore, we speculate that the inverse relation between $P A$ and these ketones appears to be specific for colonisation of $P A$ in the lungs.

Ethyl acetate was the most prominent VOC associated with $P A$ colonisation in children; its presence was inversely related with $P A$ infection. This was also found in another in vivo study that investigated VOCs emitted from sputum of 28 adult patients with either CF or non-CF bronchiectasis [14]. The absence of ethyl acetate in vitro also supports this claim [32-34]. We speculate that, while other bacterial species (E.coli or P.mirabilis) produce ethyl acetate, [32-34] PA rather degrades ethyl acetate. The degradation might be due to emittance of esterases, [35] that could potentially break down ethyl acetate. Wilhelm et al. demonstrated that esterase activity might induce a downstream signalling cascade regulating virulence-factors such as rhamnolipids [36]. We speculate that the reduction of ethyl acetate is a side product of the esterase activity that regulates virulence.

Though the strength of this study is the targeted and thus more rationale analysis of previously published VOCs in relation to $P A$, one has to realize that VOC analysis itself can be influenced by clinically unrelated processes like food intake and changes in microbiome. For example, the composite model contained limonene, which is part of citrus oils and flavouring additive [37]. The composite model also contained toluene, which can act as a source of carbon and energy for bacteria, [38] stimulating growth of $P A$, but also the growth of other bacteria influencing VOC's indirectly. Nonetheless, the links described in this study between limonene/toluene and $P A$ are replications of multiple studies $[12,14]$. Moreover, the link between toluene and $P A$ was previously established in vitro, [31,39] which does not suffer from the same limitations as breath analysis. Despite possible confounders, the validation of both in vitro and human studies in the targeted analysis increases the probability of direct chemical linkage.

The diagnostic accuracy for detection of PA that was shown in this study may be considered sufficient to influence the clinical decision making in children, though further studies are required to establish the exact role of exhaled breath analysis in disease monitoring in CF. This study resulted in an optimal cut-off with $100 \%$ sensitivity and $71 \%$ specificity. Since sensitivity is the main limitation of cough/throat swabs, [40] the most commonly used culture method in children, our results show additional value of breath screening next to current methods. The accuracy, sensitivity and specificity compare well to those found in the articles included in the literature search that also analysed diagnostic accuracy $[12,14,41]$. What is new in our study is that we validated markers that were already known to be associated with Pseudomonas while the other studies all identified unknown biomarkers. Due to high variation and lack of validation, none of the VOCs found in previous studies has moved towards clinical implementation. Using targeted analysis and performing independent validation, this study takes the next step forward.

We could not reproduce the results between children and adults in this study with single VOC analysis, though multivariate analysis provided comparable diagnostic values. Children possess a more naïve microbiota compared to adults, [42,43] increasing the need for rapid PA detection. Simultaneously, the adult CF populations is more diverse; specifically, in our cohort we identified a higher diversity in pathogen co-cultures in adults compared to children. The adult patient diversity correlates to a higher complexity in distinguishing between $P A$ positive and negative status in adults as compared to children. To further optimize the technique for adults, the sample size should be increased to allow for correction of other covariates affecting the volatile metabolome. Furthermore, this study does not make the distinction between chronic infection and early colonization; early colonization thrives clinical decision-making and antimicrobial strategy. For future studies, other covariates such as the presence of other pathogens, [44,45] antibiotic therapy, [46] and stage of infection should be considered. Although the results from this study cannot be directly extrapolated to clinic, it demonstrates that targeted VOC analysis can provide an addition to standard PA detection. A single VOC detection device potentially suffices as screening tool for $P A$ in children, while a more complex detection device might provide a suitable method of $P A$ detection or screening in adults in the future.

In summary, we performed a literature review to select VOCs with a high pre-test probability of having a good diagnostic accuracy for detection of colonisation with $P A$ and we performed a targeted analysis of the breath of children and adults with CF. Only a minority of VOCs that has been described in literature was found in exhaled breath and of those only ethyl acetate, 2-butanone, and heptane, 2,4-dimethyl were significantly different between children with and without PA. A decrease in ethyl acetate concentration in exhaled breath had a good diagnostic accuracy. For adults, a similar diagnostic accuracy required the measurement of five VOCs. These data provide evidence of clinically acceptable sensitivity values of exhaled VOCs for PA infection in CF requiring independent validation.

\section{Declaration of Competing Interests}

AHM and PB are supported by an innovation grant from Vertex Pharmaceuticals B.V.

PJS is scientific advisor and has a formally inconsiderable interest in the SME Breathomix

AHM reports grants and personal fees from GSK, Boehringer Ingelheim, and AstraZeneca, and grants from Chiesi, outside the submitted work.

\section{Acknowledgments}

We would like to acknowledge and thank all patients and parents for their time and effort put into this study. We are grateful for a research grant provided by Institut Merieux Annecy France that supported parts of this study.

\section{Funding}

This research has been supported by a research grant of the Institut Merieux. We also thank the UK Cystic Fibrosis Trust for funding this research as part of a Strategic Research Centre entitled 'Personalised Approach to Pseudomonas aeruginosa (PAPA)' via grant SRC014.

\section{Supplementary materials}

Supplementary material associated with this article can be found, in the online version, at doi:10.1016/j.jcf.2021.04.015.

\section{References}

[1] Mayer-Hamblett N, Kronmal RA, Gibson RL, et al. Initial Pseudomonas aeruginosa treatment failure is associated with exacerbations in cystic fibrosis. Pediatr Pulmonol 2012;47:125-34. doi:10.1002/ppul.21525.

[2] Nixon GM, Armstrong DS, Carzino R, et al. Clinical outcome after early Pseudomonas aeruginosa infection in cystic fibrosis. J Pediatr 2001;138:699-704. doi:10.1067/mpd.2001.112897.

[3] Ren CL, Morgan WJ, Konstan MW, et al. Presence of methicillin resistant Staphylococcus aureus in respiratory cultures from cystic fibrosis patients is associated with lower lung function. Pediatr Pulmonol 2007;42:513-18. doi:10. 1002/ppul.20604. 
[4] Amin R, Dupuis A, Aaron SD, et al. The effect of chronic infection with aspergillus fumigatus on lung function and hospitalization in patients with cystic fibrosis. Chest 2010;137:171-6. doi:10.1378/CHEST.09-1103.

[5] Esther CR, Esserman DA, Gilligan P, et al. Chronic Mycobacterium abscessus infection and lung function decline in cystic fibrosis. J Cyst Fibros 2010;9:11723. doi:10.1016/J.JCF.2009.12.001.

[6] Dalbøge CS, Hansen CR, Pressler T, et al. Chronic pulmonary infection with Stenotrophomonas maltophilia and lung function in patients with cystic fibrosis. J Cyst Fibros 2011;10:318-25. doi:10.1016/J.JCF.2011.03.006.

[7] Rosenfeld M, Emerson J, Accurso F, et al. Diagnostic accuracy of oropharyngeal cultures in infants and young children with cystic fibrosis. Pediatr Pulmonol 1999;28:321-8 5<321::AID-PPUL3>3.0.CO;2-V. doi:10.1002/(SICI) 1099-0496(199911)28.

[8] Ramsey BW, Wentz KR, Smith AL, et al. Predictive value of oropharyngeal cultures for identifying lower airway bacteria in cystic fibrosis patients. Am Rev Respir Dis 1991;144:331-7. doi:10.1164/ajrccm/144.2.331.

[9] Seidler D, Griffin M, Nymon A, et al. Throat Swabs and Sputum Culture as Predictors of P. aeruginosa or S. aureus Lung Colonization in Adult Cystic Fibrosis Patients. PLoS One 2016;11:e0164232. doi:10.1371/journal.pone.0164232.

[10] van der Schee MP, Paff T, Brinkman P, et al. Breathomics in lung disease. Chest 2015;147:224-31. doi:10.1378/chest.14-0781.

[11] Neerincx AH, Geurts BP, van Loon J, et al. Detection of Staphylococcus aureus in cystic fibrosis patients using breath VOC profiles. J Breath Res 2016;10:046014. doi:10.1088/1752-7155/10/4/046014.

[12] Savelev SU, Perry JD, Bourke SJ, et al. Volatile biomarkers of Pseudomonas aeruginosa in cystic fibrosis and noncystic fibrosis bronchiectasis. Lett Appl Microbiol 2011;52:610-13. doi:10.1111/j.1472-765X.2011.03049.x.

[13] Neerincx AH, Geurts BP, Habets MFJ, et al. Identification of Pseudomonas aeruginosa and Aspergillus fumigatus mono- and co-cultures based on volatile biomarker combinations. J Breath Res 2016;10:016002. doi:10.1088/1752-7155/ 10/1/016002.

[14] Goeminne PC, Vandendriessche T, Van Eldere J, et al. Detection of Pseudomonas aeruginosa in sputum headspace through volatile organic compound analysis. Respir Res 2012;13:87. doi:10.1186/1465-9921-13-87.

[15] de Heer K, Kok M, Fens N, et al. Detection of airway colonization by Aspergillus fumigatus using electronic nose technology in patients with cystic fibrosis. J Clin Microbiol 2015;54:569-75. doi:10.1128/JCM.02214-15.

[16] van Oort PMP, de Bruin S, Weda $H$, et al. Exhaled breath metabolomics for the diagnosis of pneumonia in intubated and mechanically-ventilated intensive care unit (ICU)-patients. Int J Mol Sci 2017;18.

[17] Boots AW, Bos LD, van der Schee MP, et al. Exhaled molecular fingerprinting in diagnosis and monitoring: validating volatile promises. Trends Mol Med 2015;21:633-44. doi:10.1016/j.molmed.2015.08.001.

[18] Hakim M, Broza YY, Barash O, et al. Volatile organic compounds of lung cancer and possible biochemical pathways. Chem Rev 2012;112:5949-66. doi:10.1021/ cr300174a.

[19] Ren CL, Hempstead SE, Accurso F, et al. Diagnosis of cystic fibrosis: consensus guidelines from the cystic fibrosis foundation. J Pediatr 2017;181:S4-S15.e1. doi:10.1016/J.JPEDS.2016.09.064.

[20] Lee TWR, Brownlee KG, Conway SP, et al. Evaluation of a new definition for chronic Pseudomonas aeruginosa infection in cystic fibrosis patients. J Cyst Fibros 2003;2:29-34. doi:10.1016/S1569-1993(02)00141-8.

[21] van der Schee MP, Fens N, Brinkman P, et al. Effect of transportation and storage using sorbent tubes of exhaled breath samples on diagnostic accuracy of electronic nose analysis. J Breath Res 2012;7:016002. doi:10.1088/1752-7155/7/ $1 / 016002$.

[22] Dieterle F, Ross A, Schlotterbeck G, et al. Probabilistic quotient normalization as robust method to account for dilution of complex biological mixtures. Application in ${ }^{1}$ H NMR Metabonomics. Anal Chem 2006;78:4281-90. doi:10.1021/ac051632c.

[23] Bos LDJ, Van Walree IC, Kolk AHJ, et al. Alterations in exhaled breath metabolite-mixtures in two rat models of lipopolysaccharide-induced lung injury. J Appl Physiol 2013;115:1487-95. doi:10.1152/japplphysiol.00685.2013.

[24] Peters AL, Gerritsen MG, Brinkman P, et al. Volatile organic compounds in exhaled breath are independent of systemic inflammatory syndrome caused by intravenous lipopolysaccharide infusion in humans: results from an experiment in healthy volunteers. J Breath Res 2017;11:026003. doi:10.1088/ 1752-7163/aa6545.

[25] Zhu J, Bean HD, Wargo MJ, et al. Detecting bacterial lung infections: in vivo evaluation of in vitro volatile fingerprints. J Breath Res 2013;7:016003. doi:10, 1088/1752-7155/7/1/016003

[26] Bean HD, Dimandja J-MDMD, Hill JE. Bacterial volatile discovery using solid phase microextraction and comprehensive two-dimensional gas chromatography-time-of-flight mass spectrometry. J Chromatogr B 2012;901:41-6. doi:10.1016/j.jchromb.2012.05.038.

[27] Bean HD, Rees CA, Hill JE. Comparative analysis of the volatile metabolomes of Pseudomonas aeruginosa clinical isolates. J Breath Res 2016;10:047102. doi:10. 1088/1752-7155/10/4/047102.

[28] Zscheppank C, Wiegand HL, Lenzen C, et al. Investigation of volatile metabolites during growth of Escherichia coli and Pseudomonas aeruginosa by needle trap-GC-MS. Anal Bioanal Chem 2014;406:6617-28. doi:10.1007/ s00216-014-8111-2.

[29] Boots AW, Smolinska A, van Berkel JJBN, et al. Identification of microorganisms based on headspace analysis of volatile organic compounds by gas chromatography-mass spectrometry. J Breath Res 2014;8:027106. doi:10.1088/ $1752-7155 / 8 / 2 / 027106$

[30] Labows JN, McGinley KJ, Webster GF, et al. Headspace analysis of volatile metabolites of Pseudomonas aeruginosa and related species by gas chromatography-mass spectrometry. J Clin Microbiol 1980;12:521-6.

[31] Zechman JM, Aldinger S, Labows JN. Characterization of pathogenic bacteria by automated headspace concentration-gas chromatography. J Chromatogr B Biomed Sci Appl 1986;377:49-57. doi:10.1016/S0378-4347(00)80760-4.

[32] Thorn RMS, Reynolds DM, Greenman J. Multivariate analysis of bacterial volatile compound profiles for discrimination between selected species and strains in vitro. J Microbiol Methods 2011;84:258-64. doi:10.1016/j.mimet. 2010.12.001.

[33] Davies TJ, Hayward NJ. Volatile products from acetylcholine as markers in the rapid urine test using head-space gas-liquid chromatography. J Chromatogr B Biomed Sci Appl 1984;307:11-21. doi:10.1016/S0378-4347(00)84068-2.

[34] Storer MK, Hibbard-Melles K, Davis B, et al. Detection of volatile compounds produced by microbial growth in urine by selected ion flow tube mass spectrometry (SIFT-MS). J Microbiol Methods 2011;87:111-13. doi:10.1016/j.mimet. 2011.06.012.

[35] Hasona A, York SW, Yomano LP, et al. Decreasing the level of ethyl acetate in ethanolic fermentation broths of Escherichia coli KO11 by expression of Pseudomonas putida estZ Esterase. Appl Environ Microbiol 2002;68:2651-9. doi:10.1128/AEM.68.6.2651-2659.2002.

[36] Wilhelm S, Gdynia A, Tielen P, et al. The autotransporter esterase EstA of Pseudomonas aeruginosa is required for rhamnolipid production, cell motility, and biofilm formation. J Bacteriol 2007;189:6695-703. doi:10.1128/JB.00023-07.

[37] Fahlbusch K-G, Hammerschmidt F-J, Panten J, et al. Flavors and Fragrances. In: Ullmann's Encyclopedia of Industrial Chemistry. Weinheim, Germany: WileyVCH Verlag GmbH \& Co KGaA; 2003. doi:101002/14356007a11_141.

[38] Prenafeta-Boldú FX, Kuhn A, Luykx DMAM, et al. Isolation and characterisation of fungi growing on volatile aromatic hydrocarbons as their sole carbon and energy source. Mycol Res 2001;105:477-84. doi:10.1017/S0953756201003719.

[39] Labows JN, McGinley KJ, Webster GF, et al. Headspace analysis of volatile metabolites of Pseudomonas aeruginosa and related species by gas chromatography-mass spectrometry. J Clin Microbiol 1980;12:521-6. http:// jcm.asm.org/content/12/4/521.short (accessed 14 Feb 2019).

[40] Seidler D, Griffin M, Nymon A, et al. Throat Swabs and Sputum Culture as Predictors of P. aeruginosa or S. aureus Lung Colonization in Adult Cystic Fibrosis Patients. PLoS One 2016;11:e0164232. doi:10.1371/journal.pone.0164232.

[41] Španěl P, Sovová K, Dryahina K, et al. Do linear logistic model analyses of volatile biomarkers in exhaled breath of cystic fibrosis patients reliably indicate Pseudomonas aeruginosa infection? J Breath Res 2016;10:036013. doi:10. 1088/1752-7155/10/3/036013.

[42] Cox MJ, Allgaier M, Taylor B, et al. Airway microbiota and pathogen abundance in age-stratified cystic fibrosis patients. PLoS One 2010;5:e11044. doi:10.1371/ journal.pone.0011044.

[43] Zemanick ET, Wagner BD, Robertson CE, et al. Airway microbiota across age and disease spectrum in cystic fibrosis. Eur Respir J 2017;50:1700832. doi:10. 1183/13993003.00832-2017.

[44] Price KE, Naimie AA, Griffin EF, et al. Tobramycin-Treated Pseudomonas aeruginosa PA14 Enhances Streptococcus constellatus 7155 Biofilm Formation in a Cystic Fibrosis Model System. J Bacteriol 2016;198:237-47. doi:10.1128/JB. 00705- 15 .

[45] Mashburn LM, Jett AM, Akins DR, et al. Staphylococcus aureus Serves as an Iron Source for Pseudomonas aeruginosa during In Vivo Coculture. J Bacteriol 2005;187:554-66. doi:10.1128/JB.187.2.554-566.2005.

[46] Neerincx AH, Mandon J, van Ingen J, et al. Real-time monitoring of hydrogen cyanide ( $\mathrm{HCN}$ ) and ammonia $\left(\mathrm{NH}_{3}\right)$ emitted by Pseudomonas aeruginosa. J Breath Res 2015;9:027102. doi:10.1088/1752-7155/9/2/027102. 